Meta

Journal des traducteurs

Translators' Journal

\title{
Clavis hermeneutica : la problématisation du traduire chez Friedrich Schleiermacher, ou l'ébauche du paradigme de la compréhension intersubjective
}

\section{Laurent Lamy}

Volume 52, numéro 3, septembre 2007

URI : https://id.erudit.org/iderudit/016746ar

DOI : https://doi.org/10.7202/016746ar

Aller au sommaire du numéro

Éditeur(s)

Les Presses de l'Université de Montréal

ISSN

0026-0452 (imprimé)

1492-1421 (numérique)

Découvrir la revue

Citer cette note

Lamy, L. (2007). Clavis hermeneutica : la problématisation du traduire chez Friedrich Schleiermacher, ou l'ébauche du paradigme de la compréhension intersubjective. Meta, 52(3), 588-602. https://doi.org/10.7202/016746ar
Résumé de l'article

Cet article se propose d'introduire à l'une des ruptures les plus significatives ponctuant notre entrée dans l'ère de la " problématicité », en considérant l'incidence déterminante de la traduction comme paradigme originel de la compréhension intersubjective dans le cadre de la théorie herméneutique développée par Friedrich Schleiermacher. Situant cette rupture dans l'horizon plus vaste qui a présidé à l'excentration du sujet rompu à sa propre finitude et entraîné une transvaluation de l'exégèse scripturaire, la problématisation du traduire engage un mouvement centrifuge et polycentrique exposant l'interprétant à une pluralité irréductible de points de vue, et à un mouvement centripète d'auto-interprétation selon lequel je ne puis comprendre un texte (un discours, une pensée) qui ne m’amène pas à me comprendre moi-même. Qui plus est, pour Schleiermacher la compréhension est l'exception,

l'incompréhension initiale la règle. Cette hétéronomie ou « loi de l'autre » est constitutive de tout acte de compréhension. C'est le défi que doit relever l'acte de traduction, qui y trouve une confirmation de son essence et l'aval de sa prérogative en tant que paradigme de la compréhension intersubjective. 


\section{Les dates}

Les dates, également utilisées sans contexte ni situation, c'est-à-dire renvoyant à tout un pan d'histoire, peuvent aussi poser des problèmes sur le plan de la compréhension d'un public qui ne connaît pas l'histoire de la société A. C'est le cas, par exemple, de 1071 (l'entrée des Seldjoukides en Anatolie), de 1299 (la fondation de l'Empire ottoman), de 1453 (la prise de Constantinople par les Turcs) et d'autres dates encore qui renvoient à des conflits et à des guerres entre beylicats turcs, etc.

Que faut-il faire de ces signes, de ces indices évoqués hors situation et hors contexte? Faut-il recourir à l'explication entre parenthèses, à la paraphrase ou à la note de bas de page pour mieux transmettre le "vouloir dire» de l'auteur? Ce sont des solutions possibles, mais c'est évidemment au traducteur d'en décider selon le public visé, car «la traduction n'est pas seulement une opération d'ordre linguistique», comme le souligne Chevrel (1989:18), «c'est aussi prendre une décision qui met en jeu un équilibre culturel et social».

\section{Conclusion}

La responsabilité du traducteur apparaît évidente et énorme, elle va même jusqu'à lui poser un problème de conscience. Il a non seulement le devoir de comprendre, mais bien au-delà de la compréhension, il a celui de connaître jusqu'à faire sien le «vouloir dire», l'idéologie de l'auteur.

Son travail de transcodage devient une véritable investigation, qui lui permet de mesurer l'impact de l'histoire, de la sociologie, de l'économie et de l'idéologie sur un texte. Il se fait alors médiateur, non sans une certaine notion diplomatique, entre deux langues et parfois entre deux cultures au sein d'une même langue.

Une problématique de traduction intraculturelle, comme c'est le cas ici entre les Turcs et les Turkmènes, laisse supposer une problématique d'autant plus difficile à gérer dans une traduction interculturelle.

Le traducteur, informé et avisé, devrait alors choisir entre explication ou interprétation, car on le sait maintenant, et cela depuis la célèbre question posée par Georges Mounin, (Traduction fidèle, mais fidèle à quoi?) qu'il a, de toute façon, un devoir de fidélité envers les deux langues.

C'est le lecteur cible qui déterminera son action. Sachant pourtant que, disposant d'un arsenal informatif abondant, le lecteur intellectuel du xxi ${ }^{\mathrm{e}}$ siècle a presque le devoir de comprendre une lecture. Le traducteur peut choisir de lui faire confiance ou de lui parler avec la voix de l'auteur tout en faisant preuve d'une sensibilité propre.
Mais si l'auteur et le lecteur lui font confiance, ceci n'en reste pas moins une mise en garde.

MÜMTAZ KaYA Université de Bilkent, Ankara, Turquie mumtaz@bilkent.edu.tr

\section{RÉFÉRENCES}

Berman, A. (1995): Pour une critique des traductions: John Donne, Paris, Gallimard.

Chevrel, Y. (1989): La littérature comparée, Presses Universitaires de France.

Glissant, E. (1996): Introduction à une poétique du divers, Paris, Gallimard.

Guiraud, P. (1983): La Sémiologie, Paris, Presses Universitaires de France.

Jammal, A. (1999) : «Une méthodologie de la traduction médicale», Meta 44-2, p. 217-237.

Ladmiral, J.-R. (1994): Traduire: théorèmes pour la traduction, Paris, Gallimard.

Lederer, M. (1986): Implicite et explicite, dans interpréter pour traduire, Paris, Didier Erudition.

Meschonnic, H. (1982): Pour la poétique II, Paris, Gallimard.

Mungan, M. (1999) : Paranın Cinleri, Istanbul, Metis yayınları.

Peeters, J. (1999): La médiation de l'étranger. Une sociologie de la traduction, Arras, Artois Presses Université, coll. «Traductologie».

SAussure, F. de (1968): Cours de linguistique générale, texte établi par Bally et Sechehaye, Paris, Payot.

Schumacher, N. (1973): "Analyse du processus de la traduction: conséquences méthodologiques », Meta 18-3, p. 308-314.

Turkmenbashi, S. (2001): Ruhnama, Achgabat.

YUAN, X. (1999): «Débat du siècle: fidélité ou recréation», Meta 44-1, p. 62-77.

\section{Clavis hermeneutica: la problématisation du traduire chez Friedrich Schleiermacher, ou l'ébauche du paradigme de la compréhension intersubjective*}

\section{RÉSUMÉ}

Cet article se propose d'introduire à l'une des ruptures les plus significatives ponctuant notre entrée dans l'ère de la «problématicité», en considérant l'incidence déterminante de la traduction comme paradigme originel de la compréhension intersubjective dans le cadre de la théorie herméneutique développée par Friedrich Schleiermacher. Situant cette rupture dans l'horizon plus vaste qui a présidé à l'excentration du sujet rompu à sa propre finitude et entraîné une transvaluation de l'exégèse scripturaire, la problématisation du traduire engage un mouvement centrifuge et polycentrique exposant l'interprétant à une pluralité irréductible de points de vue, et à un mouvement centripète d'auto-interprétation selon lequel je ne puis comprendre un texte (un discours, une 
pensée) qui ne m'amène pas à me comprendre moi-même. Qui plus est, pour Schleiermacher la compréhension est l'exception, l'incompréhension initiale la règle. Cette hétéronomie ou «loi de l'autre» est constitutive de tout acte de compréhension. C'est le défi que doit relever l'acte de traduction, qui y trouve une confirmation de son essence et l'aval de sa prérogative en tant que paradigme de la compréhension intersubjective.

\section{ABSTRACT}

This paper intends to introduce one of the most significant breaks leading to the era of "problematicity," underlining the decisive role played by translation as inchoative paradigm of intersubjective understanding in the establishment of hermeneutics by Friedrich Schleiermacher. Setting this break in the broader horizon where an excentred self can no more conceal from herself her own finiteness, and, by the same bias, giving way to a Copernician shift of value in the practice of scriptural exegesis, this topical investigation of translation displays a centrifugal and polycentred motion exposing the interpreting self to a pluralistic spectrum of points of view, as well as a centripetal movement of self-interpretation by which I cannot understand a text (a discourse, a thought) that does not yield a better understanding of myself. Moreover, Schleiermacher holds up that understanding is the exception, initial misunderstanding the rule. This heteronomy or "law of the other" belongs to the depth structure of any kind of understanding. This is the challenge that the act of translation must take up, that confirms its essence and ratifies its prerogative as paradigm of intersubjective understanding.

\section{MOTS-CLÉS/KEYWORDS}

problématicité, compréhension intersubjective, auto-interprétation, hétéronomie

J'ajoute que jamais ma recherche n'aurait pris cette tournure si je n'avais pas commencé par la traduction et constamment fait de la traduction mon but principal. Car aucune autre méthode, aucune autre discipline intellectuelle ne contraint à tenir si rigoureusement et si complètement compte de toutes les propriétés d'un texte, y compris de sa composition, de son rythme, de son ton - propriétés qui concourent toutes à lui donner son sens. Rien ne vaut l'aller et retour critique entre l'original et les versions successives de son équivalent français. Je ne suis pas loin de penser qu'une interprétation qui ne résulte pas de l'épreuve de la traduction est nécessairement subjective et arbitraire.

Jean-François Billeter

Cette remarque de Billeter, sinologue émérite, livrée en guise de prémisse à ses très belles Leçons sur Tchouang-tseu (2002: 11), est une idée qui en vérité avait commencé à germer et à frayer sa voie dans les spéculations parfois lancées tous azimuts par la première génération des Romantiques allemands.
Cette tentative de jeter les bases d'une véritable théorie critique (Benjamin 1986; Lacoue-Labarthe et Nancy 1978; Van Eynde 1997) instaurait du même coup la «scène de l'écriture » comme espace propre, privilégiant alors la forme du fragment élaboré dans une perspective encyclopédique et maniant tout aussi allègrement l'ironie et le Witz comme embrayeurs d'une autocritique de facteur exponentiel où l'œuvre génère sa propre mise en abyme. Cette percée éclair dans le firmament des idées se voulait aussi une manière de pied de nez à la façon «française» de traduire, qui imposait sans vergogne une dilution des ressources idiomatiques de l'œuvre étrangère au seul profit d'une facture dans le goût «français», songeant ici à la cuvée des «belles infidèles» dont le procédé consiste à poncer toute aspérité heurtant les attentes du lectorat indigène. La guérilla menée par les Romantiques d'Iéna se voulait certes, dans le sillage de Herder et de Goethe, un exercice conscient d'autoaffirmation de la Bildung allemande, mais, contrairement à certaines idées reçues voulant les y cantonner, les membres de cet aréopage se sont aussi distingués par une ouverture et une disponibilité sans précédent à l'égard des formes poétiques de facture étrangère. Loin de percevoir cette denrée comme une espèce de «cheval de Troie» menaçant l'intégrité à peine acquise de la langue allemande, ils y voyaient plutôt l'occasion de saisir un relais permettant une relance incessante de leurs propres ressources idiomatiques et une fécondation inespérée de leur patrimoine culturel.

Celui qui a sans doute le plus contribué à ce rééquilibrage des tensions centripètes et centrifuges qui s'exercent toujours dans le commerce entre les cultures, notamment sur le chapitre de la traduction, c'est Friedrich Schleiermacher (1768-1834). Comme le souligne Antoine Berman (1984: 227) dans L'épreuve de l'étranger, Schleiermacher est considéré comme le fondateur de l'herméneutique moderne, laquelle se distingue comme une théorie de la compréhension fondée sur l'expérience intersubjective du langage et la prise en compte de l'horizon ontologique dans lequel elle se projette et où elle s'accomplit, bref de sa situation dans l'histoire de la langue et de la culture:

L'herméneutique de la compréhension rompt avec les limites de l'herméneutique traditionnelle (essentiellement celle qui vise à ériger les règles de l'interprétation des textes sacrés) et entend se constituer comme une théorie de la compréhension intersubjective. Entendons, des processus de «lecture » qui se donnent au niveau de la communication de sujets-consciences. La compréhension d'un texte (objet exclusif de l'ancienne herméneutique) est avant tout celle du produit expressif d'un sujet. Elle est aussi celle d'un phénomène du langage objectif qui se définit moins par son auteur que par sa situation dans l'histoire de la langue et de la culture. 
Le rapport à la tradition, tant orale qu'écrite, emprunte ici des avenues beaucoup plus vastes mais se recommande aussi d'un échelonnement à la fois beaucoup plus nuancé et beaucoup plus ouvert des niveaux de signification impliqués dans la compréhension que l'exégèse qui s'en remet plus volontiers à la seule obédience à la lettre du texte sacré. Le propos de Schleiermacher s'inscrit certes dans le cadre de la tradition exégétique du protestantisme allemand qui, à n'en pas douter, constituait le terreau idéal pour l'éclosion d'une problématique entièrement renouvelée, exception faite de la rigidité du postulat lié aux principes de l'autosuffisance (sola scriptura) et de l'auto-interprétabilité (sacra scriptura sui ipsius interpretes) de l'Écriture ${ }^{1}$.

Ce n'est un secret pour personne que les Écritures, l'Ancien comme le Nouveau Testament, ont constitué la pierre de touche de l'éducation religieuse et morale de l'homme occidental. Le problème majeur qui s'est fait jour dès l'origine est celui de leur interprétation et du magistère qu'il convient de leur assortir, plus précisément à quelle instance pouvait bien être confié ledit "magistère», eu égard au conflit qui s'est très tôt ouvertement déclaré entre diverses factions quant à l'authenticité des sources et à l'établissement d'une autorité doctrinale. Problème sous-jacent: l'accès au sens du texte est conditionné par l'accès à la langue d'origine de ce texte et, par voie de conséquence, par l'élaboration d'une traduction jugée «canonique». À son tour, si l'établissement d'une version canonique pose des problèmes de méthode, il va sans dire que la sélection des leçons devant constituer le corps de l'editio princeps est déjà d'emblée problématique. On conçoit aisément que l'enjeu est de taille puisqu'il y va d'un texte dit "révélé» et qu'on ne peut relativiser outre mesure certaines décisions du «traduire» qui sont non seulement susceptibles de porter atteinte à l'intelligibilité de tel vocable ou verset, mais encore d'enrayer le processus complet de l'interprétation, compte tenu de l'interdépendance présumée de toutes les articulations du texte révélé, de son organicité.

À cet égard, j'aimerais ouvrir une parenthèse pour toucher mot de l'ascendant discret, mais néanmoins décisif, à mon sens, exercé par la tradition judaïque, plus précisément rabbinique, en matière d'interprétation (Banon 1987; Boyarin 1994; Rojtman 1987). Cet ascendant que d'aucuns sauraient toujours considérer comme nul et non avenu ou trop diffus pour constituer une réelle différence, a été largement occulté, sinon tenu sous le boisseau en raison de l'antisémitisme larvé qui a trop longtemps hanté les officines où s'exerçait le magistère catholique romain. Originellement, l'orthodoxie rabbinique répugnait à toute abondance de commentaires ou à toute forme d'exégèse systématique des versets sacrés de la Torah pour se consacrer presque exclusivement à l'affinement parfois très spécieux des préceptes rituels devant assurer la rectitude de la conduite humaine, s'en tenant pour l'essentiel à un corpus de prescriptions d'ordre éthique ou axiologique. Souvent, l'autorité doctrinale en matière de cohésion rituelle des pratiques et des obligations s'y rattachant procédait d'un assemblage hétéroclite de sources appelées à éclairer telle ou telle autre dimension de la vie en commun, sinon à valider telle ou telle autre attitude ou disposition de la vie privée devant consolider l'appartenance à la dénomination spécifique de la communauté. C’est seulement au Moyen Âge qu'un groupe d'érudits juifs tentèrent d'inculquer un ordre quelconque à ce fouillis, cet assemblage chaotique de préceptes, de lui imprimer une direction et un caractère plus canonique et universel sur la base d'une conceptualité herméneutique beaucoup plus spéculative. $\mathrm{Ne}$ s'en laissant pas imposer par la dichotomie proverbiale entre la forme et le fond, leur angle d'attaque ciblait littéralement la possibilité de débusquer et d'insuffler dans les interstices du corps pétrifié de la lettre ce feu vivant de l'esprit, cette constante vibratoire, intangible, du rythme qui épouse la pulsion de vie, assurant la prégnance des formes et conférant son unité organique au corps de la Torah. Cet art de l'interprétation se revendiquait donc d'une maîtrise consommée des formes grammaticales dont l'assise agrée à son tour l'approfondissement de la polysémie originelle des vocables hébraïques comme support ou levier de l'analogie sollicitant un jeu de consonances affines, à la faveur desquelles il était loisible de jeter des ponts entre divers segments du texte révélé, ménageant les avenues d'une élaboration allégorique ou symbolique du signifié. Cela n'allait pas sans créer des frictions, des conflits insolubles en matière d'interprétation; bref, l'orthodoxie rabbinique n'était guère disposée à lâcher la bride.

En fait, deux conceptions du magistère s'affrontaient, recoupant le conflit déjà subodoré, cet antagonisme congénital ayant presque de tout temps prévalu entre l'esprit et la lettre, le fond et la forme. Le paradoxe veut précisément que l'abstention originelle de tout commentaire de la lettre sacrée ait engendré dans la postérité, telle une onde de choc, un foisonnement exceptionnel de spéculations s'attachant à une variété prismatique d'aspects grammaticaux et d'éléments sémiques (e.g. des mots-racines servant de leit-motive) qui à leur tour pourront se prêter à diverses combinatoires au gré desquelles le tact herméneutique peut s'épanouir et prospérer, bref quérir un sens insoupçonné, un filon d'une étonnante densité sémantique sous la couche signifiante de l'énoncé. Or ce nœud gordien qui réunit, parfois à corps défendant, la composante rituelle de la Torah, qui se recommandait plus volontiers à ce stade de son évolution d'un magistère de tradition orale, et la grammaticalisation progres- 
sive de la sensibilité qui forme son réceptacle vivant, viendra plutôt renforcer, consolider l'autorité primordiale du texte révélé, lequel va en fin de compte happer dans son champ gravitationnel toute une nébuleuse de procédés littéraires, facilitant du même coup l'intégration ou l'acculturation de divers modes symboliques et constellations métaphysiques ornant le noyau vivant de la tradition scripturaire, l'insufflant et la vivifiant de nutriments spirituels inédits. C'est ce processus de métissage passablement complexe que Moshe Idel, éminent spécialiste de la mystique juive et de la littérature kabbalistique, décrit et généralise sous l'appellation d'intercorporal approach (1998: $\mathrm{x}$-xi) et que l'on peut aussi associer à ce que Daniel Boyarin désigne par ailleurs comme l'«intertextualité» caractéristique du midrash dans le cadre de l'exégèse talmudique (Boyarin 1994).

Or, c'est une révolution de pareille envergure qui s'est enclenchée dans la période de bouleversement qui a pris racine dans les prémisses de l'Aufllärung et qui a culminé avec la flambée météorique de la Frühromantik d'Iéna, pavant la voie à une conception proprement moderne de la relation au texte, révélé ou profane. Le romantisme, par exemple, c'est aussi le roman comme forme pure, parce que totalement métissée ou métissante, et autonome, parce que frappée dans son infinie générativité du sceau de la finitude, et le roman c'est la littérature qui vaut en soi, qui jouit d'une vie propre, qui a une vie à soi, puisqu'elle existe dans l'espace virtuel, interactif, de son lectorat, bref de son corps indénombrable d'interprètes qui, au demeurant, chevauchent parfois de façon inopinée le «fossé des générations». La modernité signifie aussi une problématisation vertigineuse, abyssale, de l'existence livrée à elle-même et au flux indistinct des signes qu'elle doit décoder sans jamais en épuiser le régime. Cette condition inexorable, irrémissible, est liée à la finitude du sujet, de l'agent locuteur, et, par conséquent, au caractère sui-référentiel du texte, par exemple à l'ironie critique qui le préserve de tout sommeil dogmatique et qui expose le sujet comme «centre de gravité narratif» livré au tropisme de la langue et voué à une gravitation indéfinie au sein d'une constellation hétéroclite d'éléments purement symptomatiques où la fiction identitaire est mise en abyme: on est expulsé de soi et toujours rivé à soi. Autrement dit, l'intrication de l'interprétant à l'interprété est inextricable, elle constitue la donne initiale et finale de toute existence. On songe ainsi à la stratégie pseudonymique d'un Kierkegaard, au perspectivisme généalogique préconisé par Nietzsche ou à l'«acharnement thérapeutique» auquel s'est livré Wittgenstein en soumettant les occurrences les plus prosaïques du commerce langagier à des analyses d'une précision chirurgicale: la signification n'est autre que l'usage que l'on fait de la langue.

C'est cet enchevêtrement noueux de problèmes liés à la culture du texte, qui font partie de la série de remises en question opérées tout au long du $\mathrm{XIX}^{\mathrm{e}}$ siècle et qui ont ainsi jalonné notre entrée dans la modernité, nous plongeant dans l'ère de la «problématicité» ou encore ce que d'aucuns ont désigné comme l'«ère du soupçon", que Schleiermacher a très tôt perçue comme l'enjeu principal de la crise du sens qui hantait alors les consciences et qui allait donc former le cour de la question devant mobiliser les forces vives de sa réflexion. Toute une lignée de philosophes-herméneutes va s'inscrire dans son sillage, les Dilthey, Heidegger et Gadamer, entre autres, qui vont élargir la brèche pratiquée dans l'édifice de la pensée dogmatique au gré d'analyses très approfondies du réseau de médiations marquant le passage de l'implicite à l'explicite et qui implique désormais, à la faveur de cette mutation de paradigme, un acte d'autocompréhension de la part du sujet assumant le parti pris de l'interprétation. Nous pouvons prendre la mesure de cette transvaluation et ainsi résumer tout un ensemble de formules qui répondent à autant de cas de figures du geste interprétatif, de l' «explicitation» ou de l'Auslegung selon les termes de l'analytique heideggerienne, en la réduisant à son plus simple appareil: je ne puis comprendre un texte qui ne m'amène pas à me comprendre moi-même.

Ainsi Schleiermacher est-il le théoricien chez qui va s'articuler le passage du point de vue dogmatique postulant l'autosuffisance et l'auto-interprétabilité de la lettre des Écritures à une optique proprement herméneutique qui, par une sorte de révolution copernicienne, lie d'un lien infrangible compréhension et interprétation, l'une n'allant pas sans l'autre. Ce maillage inconditionnel qui ne peut désormais faire l'économie du singulier pour qui tel ou tel autre contenu fait sens, trouve son ancrage et son espace de résolution dans l'intelligibilité pleine et entière de l'exercice du langage, dans la mesure où elle nous est accessible eu égard à la volatilité des structures émergeantes qui plongent leurs racines dans l'inconscient. Et il va sans dire, compte tenu de la pluralité irréductible des langues, que cette considération expresse de la dynamique du langage comme experimentum crucis du geste herméneutique commande une investigation minutieuse des protocoles de traduction.

Cela dit, si l'herméneutique rencontre dans l'expérience du langage sa dimension propre, en revanche c'est une dimension avec laquelle, comme le note Antoine Berman, l'homme entretient à la fois un rapport de sujétion et de liberté. C'est ce que Schleiermacher (1999: 41-43) exprime en toutes lettres dans ce passage de son essai intitulé Des différentes méthodes du traduire (Ueber die verschiedenen Methoden des Uebersezens), une conférence qu'il prononça le 24 juin 1813 à l'Académie royale des Sciences de Berlin: 
Partout où le discours (Rede) n'est pas totalement lié à des objets visibles ou à des faits extérieurs qu'il suffit d'énoncer, partout où celui qui parle pense de manière plus ou moins indépendante, et veut par conséquent s'exprimer, il se trouve vis-à-vis de la langue dans un rapport double, et son discours n'est correctement compris que dans la mesure où ce rapport l'est aussi. Chaque homme, pour une part, est dominé par la langue qu'il parle; lui et sa pensée sont un produit de celle-ci. Il ne peut rien penser avec une totale précision qui soit hors de ses limites; la forme de ses concepts, le mode et les limites de leur combinabilité sont tracés au préalable par la langue dans laquelle il est né et il a été élevé; notre entendement et notre fantaisie sont liées à celle-ci. Mais, par ailleurs, tout homme pensant librement, de manière indépendante, contribue à former la langue. [...] En ce sens, c'est la force vivante de l'individu qui produit de nouvelles formes dans la matière ductile de la langue, initialement avec son seul propos momentané de communiquer une conscience passagère; mais ces formes demeurent dans la langue, à un degré plus ou moins grand et, recueillies par des tiers, étendent leur effet formateur. [...] C'est pourquoi tout discours libre et supérieur demande à être saisi sur un double mode, d'une part à partir de l'esprit de la langue dont les éléments le composent, comme une exposition marquée et conditionnée par cet esprit, engendrée et vivifiée par lui dans l'être parlant; d'autre part il demande à être saisi à partir de la sensibilité de celui qui le produit comme une œuvre sienne, qui ne peut surgir et s'expliquer qu'à partir de sa manière d'être.

Avec Schleiermacher, comme avec Humboldt du reste, nous quittons définitivement l'univers de sens fondé sur une conception monologique du langage, qui le réduit à une portion plutôt congrue de l'expérience langagière, sa fonction instrumentale, pour accéder ainsi à sa dimension proprement dialogique et intersubjective, que Mikhail Bakhtine (Todorov 1981) a largement explorée et qui convient davantage à une «forme de vie» autonome - c'est en tant que Lebensform, "forme de vie», je me permets de le rappeler, que Wittgenstein, notamment, qualifie l'exercice du langage - définissant un milieu, une aire de jeu multidimensionnelle, pleinement temporalisée, et ainsi saturée de signes générateurs d'une multitude de renvois configurant une économie symbolique qui, en l'occurrence, se voit corrélée à un horizon historico-culturel qui forme l'univers de référence et, du même coup, la condition sine qua non de toute interaction humaine. S'il est besoin d'une interprétation ou d'une "explicitation» (Auslegung) des contenus immédiatement accessibles à la compréhension (Verstehen), c'est qu'on rencontre incessamment des facteurs d'opacité, des éléments litigieux, des énoncés conflictuels ou contrefactuels, sinon des configurations de sens elliptiques ou purement suggestives qui requièrent un supplément d'information et, par conséquent, justifient le recours à l'interprétation.

Ce dont Schleiermacher prend conscience, en fin de compte, c'est rien moins que la condition même de la modernité, sa dimension critique: le réquisit d'une ontologie plurielle confrontée à une diversité de points de vue qui, non seulement se réfléchissent dans le langage, mais y trouvent leur consistance, car il s'agit bien d'un milieu, d'un médium, d'une matrice vivante qui déborde largement la gestion formelle des unités de sens entrant dans la composition de tel ou tel autre énoncé. Comme le souligne Peter Szondi (1975: 297) dans la brillante étude qu'il a consacrée à l'herméneutique de Schleiermacher :

L'important n'est donc pas l'interprétation de passages particuliers, mais la saisie du texte parlé ou écrit à son origine, à partir de la vie individuelle de son auteur: la parole et l'écrit sont alors saisis à la fois comme «jaillissement d'une portion de vie» et comme acte, cessant ainsi d'être de simples documents pour devenir expression active et actuelle de la vie.

C'est pourquoi j'ai évoqué plus haut la notion de "problématicité », dans la mesure où l'aire de jeu des interprétations, qu'on devine aussi bien être concertantes que grosses de différends de toute sorte, doit demeurer ouverte, ne serait-ce qu'elle implique et sollicite divers intervenants: auteur, traducteur, lecteur, interprète, critique. Autant la trace écrite ou la vive parole doit témoigner d'un acte de discours ou d'une prestation de sens jaillissant de la vie de son auteur, autant celui vers qui est dirigé cet acte, même à l'insu de son destinateur ou de son destinataire, doit-il le soumettre aux critères qui gouvernent sa propre existence. La distance critique aussi bien que l' "épiphanie» du sens, l'éclairage soudain auquel il est promu, ne sont plus l'«affaire» d'un magistère réglé déterminant de façon préjudicielle les paramètres de l'interprétation, mais d'une interaction dialogique entre les parties en cause. Le processus de traduction rejaillit sur tous les plans de l'existence. Et c'est précisément le point de vue qu'adopte Schleiermacher au départ de son essai sur les «différentes méthodes du traduire». Il met directement en évidence ce premier niveau de difficulté qui frappe le locuteur dans le rapport à sa propre langue, qui n'est en fait que l'indice minimal d'une problématique du traduire qui s'étend sur tout un spectre de situations interlocutoires, qui en appelle chez Schleiermacher à une réflexion sur la traduction "généralisée». Comme le note Berman (1984: 232):

[...] il y a partout «traduction » là où nous devons interpréter un discours: qu'un étranger nous parle dans une langue qui n'est pas la nôtre, qu'un 
paysan nous interpelle en patois, qu'un inconnu tienne des propos que nous comprenons mal, ou que nous nous penchions sur des propos que nous avons autrefois tenus, mais qui nous paraissent désormais obscurs... dans tous les cas nous sommes conduits à un acte de «traduction" - le plus difficile n'étant pas forcément celui qui concerne une langue étrangère.

Le passage de la traduction "généralisée», qui est implicite à tout acte de communication, à la traduction « restreinte» en appelle chez Schleiermacher à la distinction entre l'interprétariat, qui concerne la situation orale ou "performative» du discours, et le champ du traduire qui se rattache plus volontiers à la trace écrite, laquelle dénote la présence d'une empreinte subjective, par contraste avec le champ de l'interprétariat, celui des « discours où le langage tend à devenir pure désignation sans épaisseur. Ici, non seulement celui-ci est simplifié à l'extrême, mais il n'a pas de valeur en lui-même, il n'est que le véhicule indifférent d'un contenu» (Berman 1984: 233). Ce qui est en jeu ici c'est un paradigm shift au sens même où l'entend Thomas Kuhn, à savoir une mutation du paradigme du langage où ce dernier n'est plus seulement relégué à la fonction instrumentale le subordonnant à un pôle de la représentation, celui de l'intention signifiante, mais comme vecteur de l'expression où il engendre du sens. Et c'est là, en fait, une caractéristique de l'époque, puisque l'herméneutique de Schleiermacher prolonge la vision de l'Athenäum, l'organe de diffusion des adeptes de la Frühromantik regroupés autour de Friedrich Schlegel à Iéna et dont Schleiermacher était un membre actif, corrélativement à la visée anthropologique développée par Wilhelm von Humboldt à la faveur de ses recherches sur l'essence et la fonction du langage. Désormais, comme l'écrit Michel Foucault (1966: 301), le langage est censé «s'enraciner non pas du côté des choses perçues, mais du côté du sujet en son activité».

La traduction proprement dite répond à ce nouvel "état de choses", qui sollicite une conscience accrue de l'historicité fondamentale sous-tendant l'ensemble des pratiques signifiantes qui contribuent à l'élaboration de l'univers de référence d'une communauté de locuteurs. Or, l'amplitude de l'écart déterminé par l'épaisseur du temps qui sépare deux langues, sans compter la distance géographique, ou encore deux "états» de la même langue, et les intériorités respectives ou, si l'on préfere éviter cette fiction métaphysique, les «centres de gravité narratifs » de l'auteur opérant dans la langue-source et du lecteur nourrissant des attentes dans la langue-cible, tout ce décalage constitue précisément l'objet répondant à la visée implicite de la traduction. Celle-ci, il va sans dire, doit déjà négocier avec le coefficient de difficulté affectant le mode de visée implicite au texte d'origine, lequel répond à l'équation complexe formée par l'exercice arbitraire d'une subjectivité qui s'exprime et, par ailleurs, l'ordre objectif des contenus disponibles dans la juridiction sémantique de sa langue, s'agissant alors de ménager une voie de passage vers la langue propre du pôle réceptif qui comporte lui-même un indice de difficulté non moindre lié à l'espace de résolution des nébuleuses sémantiques qui ne peuvent manquer de s'avérer préjudicielles à toute translation symétrique. L'asymétrie des langues, qui est la résultante d'une évolution historique composant avec une diversité de facteurs, d'acculturation ou d'isolement par exemple, est incorrigible.

La problématique qui s'annonce est fort bien définie par Schleiermacher: elle tourne autour d'une conception de la traduction comme processus de rencontre intersubjectif, livré comme tel au dilemme, ou plus simplement à l'alternative entre une traduction ethnocentrique ou non ethnocentrique: ou le lecteur est amené vers le texte d'origine, ou ce dernier est tiré vers le lecteur. La traduction devient ici un chapitre de la compréhension et l'enjeu en est précisément la possibilité de produire une traduction authentique (eigentlich). Deux procédés sont examinés par Schleiermacher: la paraphrase et l'imitation (Nachbildung).

La paraphrase, écrit-il, veut «éliminer l'irrationalité des langues, mais de façon purement mécanique» (Schleiermacher 1999: 45). La pénurie de termes appropriés est palliée à l'aide de «compléments amplifiants ou limitatifs »; bref, la paraphrase «opère avec les éléments des deux langues comme s'il s'agissait de signes mathématiques qui, par addition ou soustraction, pouvaient être ramenés à une valeur égale, et de cette façon, ni l'esprit de la langue transformée, ni l'esprit de la langue d'origine ne peuvent se manifester» (1999: 46). La Nachbildung, l'imitation ou «recréation", en revanche, «se plie à l'irrationalité des langues» et ne connaît d'autre aménagement que celui qui consiste à «élaborer une reproduction" où l'on doit renoncer à retracer l'original, s'agissant tout simplement de sauver l'unicité de l'impression suscitée chez les lecteurs d'origine dans la facture de l'œuvre traduite. Pour Schleiermacher ces deux avenues, qui sont fallacieuses, sinon carrément stériles, ne figurent ici que "comme des points-limites du domaine qui nous concerne (nur als Grenzzeichen für das Gebiet)».

Première constatation eu égard à la possibilité d'en venir à une traduction qui sache approcher l'esprit et la lettre du texte d'origine et les restituer dans la langue-cible sans trop faire violence aux attentes du lecteur indigène: on ne saurait produire une version telle que l'auteur l'aurait écrite s'il eut été un locuteur de la langue d'accueil, parce que cette perspective nie le rapport profond qui lie cet auteur à sa langue propre. Qui nie l'autre se nie soi-même. En 
revanche, seule une langue maternelle suffisamment développée, pour ainsi dire parvenue à sa maturité, bref à un stade critique de son affirmation, est en mesure d'accueillir en son sein une œuvre étrangère et de lui rendre justice. C'est précisément le processus qui était en train de s'enclencher à l'époque où Schleiermacher réfléchissait à tous ces problèmes l'émancipation de l'aire d'influence de la culture française, ce classicisme corseté qui ne savait se livrer qu'à une opération de Nachbildung ethnocentrique. À la suite du précédent créé par Luther au Xvi ${ }^{\mathrm{e}}$ siècle, Goethe à Weimar et les membres de l'aréopage d'Iéna regroupés autour de l'Athenäum, Schleiermacher revendique l'élaboration d'une Bildung marquée par une liberté respectueuse de l'étranger, mais qui assume en même temps son identité propre. Autrement dit, "présenter l'étranger dans la langue maternelle», selon l'expression de Schleiermacher, n'est pas la conséquence d'une opération toute formelle, c'est accepter, comme le précise Berman (1984: 240-241) que la langue maternelle soit:

[...] élargie, fécondée, transformée par cet «étranger », accepter la «nature médiatrice» de celui-ci, c'est là un choix qui précède toute considération étroitement méthodologique. Or, ce choix, c'est toujours le choix d'une méthode, d'un met'hodos, d'un chemin, c'est toujours le tracé d'un champ à parcourir, jalonner, cultiver. Et c'est le mérite de Schleiermacher que d'avoir présenté ce choix comme celui de l'authenticité, en le confrontant à un autre choix possible, celui de l'inauthenticité. Car ces deux concepts unissent la dimension éthique et la dimension ontologique, la justice et la justesse.

Ce choix éthique commande une perspective plus large qui en appelle à la traduction massive d'œuvres de source et de provenance les plus diverses, de façon précisément à élire un espace colloquial qui agrée l'accroissement et l'intensification des ressources expressives de la langue d'accueil. La visée de Schleiermacher est stratégique: il faut un Sprachgebiet particulier pour les traductions, un domaine de prédilection, un champ qui lui soit propre à l'intérieur du champ culturel, pour que l'étranger puisse remplir sa fonction médiatrice et ainsi mobiliser des ressources insoupçonnées de la langue maternelle, éveiller et féconder les éléments qui y sommeillent encore à l'état de latence.

Schleiermacher est lui-même un traducteur émérite. À un âge encore précoce, il traduira les chapitres 8 et 9 de l'Éthique à Nicomaque d'Aristote (1789); mais sa contribution majeure, qui compte parmi les plus belles réussites de la langue allemande, c'est sa traduction complète (à l'exception du Timée et des Lois) des œuvres de Platon, entamée avec son ami Friedrich Schlegel, qui en avait pris l'initiative en 1798, et qu'il dut achever seul. Ce labeur va le tenir en haleine pendant plus de vingt ans; il sera étayé d'une copieuse introduction et d'un appareil critique où Schleiermacher, à l'appui de considérations historiques, philologiques et proprement herméneutiques, se livre à une reconstruction systématique des dialogues platoniciens, visant l'obtention d'une editio princeps la plus fiable possible pour ainsi s'assurer d'une traduction avalisée par les sources les mieux informées. Ce travail colossal, depuis constamment réédité, fait toujours autorité (Schleiermacher 2004).

Sa conception de la traduction est indissociable de l'élaboration d'une herméneutique qui est ellemême assortie à une dialectique et à une éthique. Comme nous le rapporte Christian Berner dans son introduction aux Différentes méthodes du traduire, «l'art de comprendre, c'est-à-dire l'herméneutique générale, ébauchée dès 1805 , reçoit sa première forme systématique en 1809-1810; l'éthique est reprise et systématisée en 1812-1813 et la dialectique, enseignée dès 1810-1811, trouvera sa forme essentielle en 1814-1815» (1999: 12). Du reste, eu égard à la gestation et à l'agencement plus systématique de la pensée critique qui est en train de s'ébaucher chez Schleiermacher, on ne saurait sans en trahir l'esprit et la lettre canoniser l'herméneutique comme discipline maîtresse à laquelle seraient tout simplement subordonnées la dialectique et l'éthique. C'est plutôt le contraire qui prévaut comme le souligne Denis Thouard (2002: 18, note 7), Schleiermacher, écrit-il,

[...] a enseigné en fait alternativement l'herméneutique "générale» (comme en 1809-1810), essentiellement subordonnée à la logique (la Dialectique), et l'herméneutique spéciale du Nouveau Testament en s'appuyant au début sur les traités d'Ernesti et de Morus, dont le caractère «littéraire» est second, mais effectivement présent. La portée philosophique de l'herméneutique ne peut guère être appréciée chez lui indépendamment de sa Dialectique. La place de l'herméneutique dans sa philosophie a été manifestement surévaluée par les lectures cherchant à faire de lui un ancêtre de l'herméneutique philosophique.

Cela dit, force est de constater que le projet de Schleiermacher se définit d'emblée comme une entreprise essentiellement spéculative, c'est-à-dire soucieuse de se maintenir à un niveau très élevé de généralité, par contraste avec le chantier développé entre autres par Wilhelm von Humboldt, qui s'efforçait de rendre fusible une masse hétérogène d'éléments colligés en puisant à une diversité foisonnante de sources débordant largement le bassin des langues indo-européennes. Enfin, faut-il le rappeler, les vues de Schleiermacher répondent pour l'essentiel aux prérogatives d'une pensée d'inspiration religieuse, une pensée innovatrice certes, mais qui demeure celle d'un théologien protestant et dont les fils enchevêtrés tissent la trame d'une interrogation 
qui en première et dernière analyse se veut d'ordre éthique (Pym 1997).

La conférence sur la traduction (1813) s'inscrit donc dans le cadre conceptuel d'une herméneutique déjà passablement élaborée, non seulement dans ses grandes lignes mais eu égard aux dimensions de sens ouvertes par la responsabilité qui incombe à une interrogation qui entend saisir le comment et le pourquoi de notre être au monde. La traduction est partie prenante de ce questionnement, dont Christian Berner déploie ainsi les trois volets (Schleiermacher 1999: 12):

- Pour l'herméneutique, traduire est un cas particulier de l'acte de comprendre dont il differe par le degré et les principes herméneutiques s'appliquent à la théorie de la traduction.

- Pour la dialectique, penser et parler sont intimement liés et la traduction est indispensable au dialogue qui construit le savoir en édifiant un système de concepts correspondant au réel.

- Pour l'éthique, la traduction conditionne les échanges et la communication, fondateurs des communautés qui sont l'objet de l'éthique.

La conférence de Schleiermacher comporte un élément polémique dirigé à l'encontre de Friedrich August Wolf (1759-1824), qui était à la fois le maître, le modèle et l'adversaire de Schleiermacher en matière de philologie. Or, Wolf justement n'avait guère prisé les traductions de Platon proposées par Schleiermacher, les considérant tout simplement "illisibles». Ici Schleiermacher vise Wolf et l'Aufklärung dont il était l'un des tenants, lorsqu'il rejette l'idée que les langues sont des réserves de signes interchangeables, "comme s'il s'agissait de signes mathématiques qui, par addition et soustraction, pouvaient être ramenés à des valeurs égales » (Schleiermacher : 47). Pour Wolf, il suffit d'augmenter ou de diminuer le nombre de signes pour obtenir une version approximative qu'il estime viable, c'està-dire une détermination conceptuelle à peu près équivalente dans les deux langues. Schleiermacher répudie pareillement la paraphrase et l'explication historique se présentant en guise de commentaire censé combler l'écart entre le passé et le présent, n'étant qu'une démultiplication superflue de signes destinés à recouvrer le sens que l'auteur aurait originellement imprimé à son texte (Neschke 1990: 257-258). Schleiermacher est donc désireux de quitter le terrain de la «traduction immédiate» (le Dolmetschen, terme ancien venu d'Asie et transplanté en allemand à partir du turc et du magyar), le «truchement» dont la visée est celle d'une simple translation, d'une commutation de valeurs linguistiques équivalentes, pour atteindre le domaine (Gebiet) de la traduction authentique (eigentlich) qui, comme le souligne Christian Berner, fait de la traduction « un paradigme, un modèle où conver- gent comme en un foyer les perspectives essentielles de sa philosophie» (Schleiermacher 1999: 14).

D'abord, pour Schleiermacher la différence entre l'acte de comprendre (verstehen) et l'acte de traduire (übersetzen) n'est qu'une différence de degré. Ensuite, on ne traduit que du discours (Rede), bref l'expression d'une pensée structurée par la transitivité de son intentio, qui est toujours dirigée vers un pôle interlocutoire, qu'il soit effectivement présent ou qu'il opère in absentia dans la forme même de son énoncé. Le rapport implicite, préalable, inscrit en filigrane dans tout acte de langage, est celui d'un dialogue (Gespräch). La langue est un milieu, plus précisément un « universel singulier». C'est-à-dire un code général (sémantique et syntaxique) actualisé par un individu locuteur dont l'acte de parole-Schleiermacher innove ici en forgeant le terme Sprechakt - individualise la langue.

Le «traduire» déborde ainsi la seule compétence ou expertise linguistique, son actualisation engage mon rapport à autrui et au monde ainsi que le rapport à la langue où s'inscrit ce rapport. Je ne traduis pas une langue mais un acte de discours, et celui-ci commande un acte de compréhension inscrit dans la virtualité même des ressources expressives imparties par l'usage d'une langue que je partage avec d'autres locuteurs et dont les contenus et les visées implicites sont susceptibles d'être traduits, donc partagés avec les locuteurs d'une autre langue. Ainsi que le stipule Christian Berner (Schleiermacher 1999: 16) c'est

[... ] sous cette forme que la pensée assume l'exigence d'universalité que Friedrich Schlegel résumait dans la formule du gemeinschaftliches Selbstdenken qu'il avait élaborée en lisant Platon et expérimentée quelque temps avec Schleiermacher à Berlin à partir de la fin de l'année 1797: penser, c'est «penser par soi-même en commun avec le autres ». Il ne fait pas de doute que sous cet angle, traduire est indissociable de la pensée, l'autre devant pouvoir traduire ma pensée de même que je dois être capable de transposer la sienne pour comprendre vraiment.

Mais ce que Schlegel appelle de ses vœux, à savoir «le développement vivant du penser par soimême en commun", qui emprunte la forme d'un dialogue (Gespräch) implicite de la pensée où le pôle de l'altérité interlocutoire est pour ainsi dire préstructuré, n'est pas pour autant acquis. Il y a un effort de compréhension - songeons aux divers aléas, méprises et tractations interprétatives auxquels furent souvent exposés les Bronislav Malinovski, Franz Boas, Margaret Mead ou Claude Lévi-Strauss -, un mouvement vers l'autre qui est requis. Cette hétéronomie ou «loi de l'autre» n'est pas du tout fortuite, mais plutôt constitutive de tout exercice de compréhension. Bref, elle est structurante, sinon préstructurée dans la gestation même des grammaires qui 
gouvernent jusqu'aux délibérations les plus intimes de l'agent locuteur. C'est là le constat dont part Schleiermacher, qui en tire une leçon exemplaire, une thèse radicale, éminemment moderne, comme le note Christian Berner: "la compréhension est l'exception, l'incompréhension initiale la règle». Il ne s'agit pas d'un simple cas d'«opacité référentielle», tel que l'a supputé Quine (1969) en élaborant sa thèse sur l'indétermination radicale de la traduction, bien au contraire, «la non-compréhension - ou la mécompréhension - est à l'origine de tout processus de compréhension et d'interprétation " (Schleiermacher 1999: 17).

Cette prémisse incendiaire n'est pas non plus sans préfigurer une notion plus récente introduite dans le cadre de la pensée analytique de tradition anglo-américaine, soit le principle of charity, mobilisé aussi bien par Donald Davidson que par Quine (Delpla 2001). Ramené à sa plus simple expression, tel que le stipule Jean Greisch (2000: 101), ce principe consiste à

[...] supposer qu'il n'y a pas d'interprétation possible d'un énoncé si l'on n'est pas capable d'identifier les «attitudes propositionnelles » d'un locuteur. Une théorie de l'interprétation qui pose une corrélation étroite entre la croyance et la signification doit parier sur le fait que le locuteur «sait» ce qu'il dit, qu'il croit que ce qu'il dit est vrai et qu'en plus, la majeure partie de ce qu'il dit est vrai.

Cette triple créance accordée à l'interlocuteur, même dans le cas où il formule une simple croyance, qui est sans doute l'une des «attitudes propositionnelles» les plus fréquemment affichées, est intimement liée à la situation initiale qui prévaut dans l'interaction entre locuteurs, ou auteur et lecteur, texte et interprète, et dont Schleiermacher estime qu'elle comporte toujours un indice d'opacité, de mécompréhension. La possibilité même d'une " traduction radicale» des stimuli sensoriels ou des sense data, que l'on loge tout naturellement dans la sphère de l'«expérience privée» (Wittgenstein 1982), en un énoncé crédible est tributaire de ce triple pari, de la présomption de vérité (de sincérité) qui accueille l'assertion de mon interlocuteur. Dans l'horizon plus vaste de l'éthique, de l'obligeance implicite qu'il m'incombe d'assumer face à l'interpellation de l'autre homme et qui touche par ce biais à la dimension anthropologique, ce principe s'oppose à toute considération d'une pensée dite "primitive" ou «prélogique» qu'on imputerait à tout interlocuteur dont $\mathrm{l}^{\prime}$ «attitude propositionnelle» ne répondrait pas aux critères de la rationalité développée en Occident (Laugier 2001; Berner 2001). Personnellement, quoiqu'il ne soit pas lieu d'en débattre ici, j'estime que le choix du terme "charité» n'est pas des plus heureux, dans la mesure où il pourrait connoter une certaine condescendance, une forme de magnanimité de la part de l'interprétant qui tente d'appréhender et de distiller, même à son insu, l' « exotisme » de la parole étrangère. Quoi qu'il en soit, il y a toujours un «reste», un «résidu » de mécompréhension ou d'«opacité référentielle» si l'on peut dire, qui creuse l'abîme entre les deux pôles d'un acte de langage: l'équivoque ne peut jamais être complètement levée et cette inscrutabilité foncière remonte sans doute à l'«enfance de l'art » en matière d'expression. Et il n'est pas sûr que l'asymétrie ou la part d'équivoque ressentie au contact d'une culture exogène soit plus prononcée que celle qui sévit déjà dans la communication entre locuteurs d'une même communauté linguistique. Bref, le rapport à notre propre langue, même dans sa teneur la plus prosaïque, recèle toujours de l'Unheimlich, une «inquiétante étrangeté» dont la traduction révèle l'amplitude dans la sollicitation de l'étranger qui vient assiéger les ressources d'une expression que l'on avait jusque lors le loisir d'estimer ouverte à toute possibilité. La traduction n'est vecteur de sens dans un horizon ouvert à l'infini que sous condition de s'y révéler aussi un marqueur de notre finitude.

Schleiermacher a lui-même pris la mesure de ce hiatus en considérant à rebours l'étonnante plasticité exhibée par le jeune enfant dans la période où il fait l'apprentissage, pour le moins prolifique, du fonctionnement et de la gymnastique l'habilitant à solliciter les éléments génériques de sa langue maternelle. Dans le second Discours académique, prononcé en 1829 , où il expose ses vues sur l'herméneutique devant l'Académie des Sciences de Berlin, il n'hésite pas en effet à marquer son étonnement (Schleiermacher 1995: 172):

Cette première activité dans le domaine de la pensée et de la connaissance me paraît toujours si digne d'étonnement qu'il me semble que nous ne sourions aux fausses applications des éléments linguistiques recueillis que font les enfants, et à vrai dire souvent par simple excès de logique, qu'afin de nous consoler ou de nous venger de cet excédent d'une énergie que nous n'arrivons plus à mettre en œuvre.

On le sent bien, pour Schleiermacher ce frayage intempestif et pourtant commun à tous les individus de notre espèce, où le babil laborieux du petit d'homme se métamorphose et se surprend à apprivoiser le clair vocable et le ressac encouru dans la réponse d'autrui à telle ou telle autre intonation, n'est pas que vague gymnopédie destinée à distraire l'adulte chez qui cet élan s'est depuis longtemps brisé et emmuré dans le mutisme et la formule creuse. Pas de doute que notre homme eut été fasciné par les travaux de Chomsky, ce qui plus est, ainsi que l'observe Jean Greisch (2000: 107), un peu plus loin dans le texte Schleiermacher explique qu'à ses yeux, 
[...] l'herméneute adulte le mieux exercé se trouve toujours logé à la même enseigne que l'herméneute en culottes courtes qu'est l'enfant: la situation fondamentale demeure inchangée, pour autant que toute interprétation est une entreprise risquée qui requiert une "témérité divinatoire» dont aucune technique et méthode ne viendront à bout.

Mais cette «témérité divinatoire» est certes aussi l'apanage de la traduction lorsqu'elle confronte l'amplitude des interférences d'ordre sémantique ou syntaxique ou la simple discordance entre les extensions respectives des tropes dans la translation de la langue-source à la langue-cible. Bref, la langue «maternelle» nous laisse parfois orphelins, comme démunis face à une tournure dont le tropisme nous entraîne dans une contrée étrangère de l'expression où les repères familiers s'estompent au profit d'une configuration de sens totalement étrangère. Or, c'est précisément ce qui justifie, à maints égards, l'acte de traduction, car si l'incompréhension est initiale, à la rigueur même cooriginaire à l'exercice de toute pensée, elle n'est cependant pas définitive, en sorte que nous sommes tous investis, sinon conviés, bon gré mal gré et à divers degrés certes, comme mandataires ou avoués d'une "communauté idéale de communication» (Habermas). En ce sens, comme l'avait déjà intuitionné Friedrich Schlegel, cet élan vers la communauté, la mutualité de son compagnonnage, compte tenu de l'inadéquation foncière régissant l' "entre-deux » des langues qui témoignent de la diversité des cultures ornant le microcosme humain, frappant originellement toute translation d'une asymétrie incorrigible, détermine un rapport ouvert, selon lequel «chaque traduction est une tâche indéterminée, infinie» (Thouard 1996: 214).

La facture d'une œuvre dans une langue et sa traduction dans une autre s'inscrivent dans des réseaux conceptuels distincts. L'épreuve du temps et la constitution d'horizons sociohistoriques donnés introduisent un décalage dans la translation des formes langagières et dans leur aptitude à véhiculer des concepts. L'incommensurabilité des paradigmes en science, fermement établie par Thomas Kuhn au $\mathrm{xx}^{\mathrm{e}}$ siècle, est encore plus éloquente et solidement implantée dans le commerce entre les langues et n'est du reste pas étrangère à celle qui est relevée par Kuhn. Inutile par ailleurs de s'étendre sur les avatars aussi bien que le fond indéniable de vérité qui ressortissent à la thèse de Sapir-Whorf sur la relativité des cultures en rapport avec les usages respectifs de l'une ou l'autre langue ${ }^{2}$. Il ne s'agit pas pour autant d'abdiquer toute possibilité de ménager des «vases communicants», des voies de passage entre les " archipels de la différence» : tout brouillage définitif entre les langues est exclu; la perte ou l'entropie présumée dans la translation entre les nébuleuses sémantiques corrélées aux divers idiomes et dialec- tes est aussi une vue de l'esprit confiné aux paramètres d'une certaine rationalité, d'une forme plutôt abstraite de traitement du flux énergétique de la parole qui voudrait que tout ce qu'il nous est loisible de penser puisse ou doive même s'énoncer clairement.

Quoi qu'il en soit, Schleiermacher était pleinement conscient de l'ascendant exercé par cette incommensurabilité ou asymétrie de principe, lui pour qui «l'enjeu de l'acte de traduire se situe au niveau de la pensée en général et de son rapport avec le réel, ce qui engage une conception du sens» (1999: 20). En retour, l'aptitude à générer du sens, à en accroître la portée, en appelle à un effort concerté des forces vives de la Bildung, la genèse d'une culture vivante qui s'inscrit d'emblée dans une dimension historio-critique se prêtant à divers degrés tantôt à une épiphanie tantôt à une éclipse du sens. Ce sens épouse une diversité incirconscrite de vecteurs qui lient l'éclosion et le déclin d'une langue à son histoire; «la langue étant un être historique, écrit Schleiermacher, il ne peut y avoir un authentique sens de celle-ci sans le sens de son histoire» (1999: 57). Schleiermacher est conscient aussi qu'il faut atteindre à une masse critique de traductions pour qu'un tel élan puisse entraîner avec lui l'ensemble de la culture d'un peuple et l'instaurer comme médiatrice idéale des ressorts de la connaissance et de l'expression artistique. La culture de l'Allemagne post-romantique serait parvenue à ce stade critique qui s'inscrit dans la mouvance d'une Bildung qui héberge dans son amplitude cette teneur de sens élargie au contact de l'étranger qui entre désormais dans la configuration de l'axe dialogique qui lie individu et communauté et les projette dans un horizon ontologique répondant à une déclinaison décisive de l'histoire universelle de la culture.

Cette dimension culturelle de la traduction correspond pour Schleiermacher à ce qu'il désigne comme «éthique» et qui n'est autre pour lui que le levier de la science du «développement de l'esprit» (Geistesentwicklung), qui se veut éminemment respectueuse de la culture de l'étranger mais sans sacrifier pour autant l'apanage de la langue natale, de l'enracinement - dans une culture plutôt que dans une sol ou une ethnie, dirais-je plus volontiers - qui seul sait agréer une véritable Einfühlung, une forme naturelle d'empathie qui se prête à l'hospitalité et à la convivialité. Le cosmopolitisme inhérent à l'entreprise de traduction s'enracine dans la singularité de l'universel incarné dans l'usage toujours difficile de ce qui nous est le plus propre, à tel point que nous finissons par en ignorer la dynamique. C'est pourquoi, comme l'a bien pressenti Hölderlin, l'épreuve de l'étranger n'est autre que l'apprentissage du propre. Dans une lettre adressée à Böhlendorff et datée du 4 décembre 1801, il affirme que «ce qui est propre doit tout aussi bien être appris que ce qui 
est étranger», car «le libre usage de ce qui nous est propre est ce qu'il y a de plus difficile» (Hölderlin 1967: 640).

Or, la traduction a cette vertu de nous inscrire dans un rapport insigne avec notre propre langue dont la singularité en appelle à une «fusion des horizons » qui, quoique jamais totalement réalisée, porte dans sa virtualité même le sens véritable de l'universalité. Chaque langue est un être historique, donc vivant ou ayant connu une $\mathrm{vie}^{3}$, bref une forme de vie autonome appelée à se développer, à connaître son floruit, à encourir certaines greffes tantôt salutaires tantôt funestes car la parasitant ou la forçant à s'étioler au profit d'une lingua franca impérialiste qui pourra réduire son usage à une portion très congrue, sinon la reléguer à une fonction satellite, jusqu'au point de l'éclipse définitive, à l'état de vestige ou de condition fossile. En tous les cas, la singularité de chaque langue est une donnée dont on ne peut faire l'économie pour accéder à l'universel. Sinon l'universalité n'est que pure abstraction ou vœu pieux répondant tout au plus à un œcuménisme de pacotille.

L'impossibilité à laquelle accule parfois l'obligation d'en découdre avec l'élément étranger dans la traduction est la possibilité même de la traduction. Autrement dit, l'impossibilité de la traduction est la condition de la traductibilité de toute œuvre comme de tout code. Cette impossibilité qui tient en son suspens toute possibilité dans l'ordre de l'expression, qui la potentialise en l'assignant à sa tâche proprement infinie, est à la mesure de notre condition, la finitude assumée dans l'ampleur concrète des virtualités frayant leur voie dans les ressources expressives d'une langue à une époque donnée de son évolution (Budick et Iser 1996).

Cet «état de choses» est la donnée constitutive de l'herméneutique comme art ou science de l'interprétation sollicitée par l'ensemble des pratiques signifiantes à la faveur desquelles nous tentons d'apprivoiser les tenants et aboutissants de notre condition. Le terme "herméneutique», dont la notion aurait été introduite comme telle au XVII ${ }^{\mathrm{e}}$ siècle, prenant le relais de la notion théologique d' "exégèse", dérive du terme grec hermeneia, et de son excroissance hermeneutikè, où l'on peut facilement décrypter le nom du dieu grec Hermès, messager des dieux, figure emblématique (avec son caducée) de la communication et des échanges commerciaux. C'est à la croisée des chemins de l'Aufklärung et du Romantisme allemand que l'herméneutique s'est développée comme discipline autonome exclusivement concernée par la compréhension médiate ou l' «explicitation» (Auslegung) des contenus de sens visés par toute expression orale ou écrite. Schleiermacher est responsable de l'unification de ce projet qui touche à diverses dénominations « régionales» de l'interprétation, par exemple l'exégèse théologique, la philologie, la jurisprudence et les sciences historiques, sans compter le vaste domaine de spécialisation de la traduction, de la terminologie et de la lexicographie. La thèse centrale de Schleiermacher veut que l'interprète ou l'herméneute réactualise à la faveur d'un acte réflexif et imaginatif le sens du texte ou du discours, de façon à exhiber et à clarifier les intentions véritables de l'auteur, à la lumière des éléments qui forment la trame de sa vie et des conditions sociohistoriques qui ont vu surgir l'œuvre. Hans-Georg Gadamer, maître d'œuvre de la philosophie d'inspiration herméneutique au $\mathrm{xx}^{\mathrm{e}}$ siècle, cerne bien l'impératif qui gouverne cette démarche: «La compréhension est la répétition reproductive de la production spirituelle originelle sur la base de la congénialité des esprits» (1996a: 93-94).

Le projet de Gadamer est sans conteste, par le relais de l'analytique existentiale de Heidegger, l'excroissance la plus aboutie d'une tradition de pensée qui s'est engagée chez Wilhelm Dilthey (1833-1911) et qui visait à fonder ce qu'il désignait alors comme les Geisteswissenschaften, les "sciences de l'esprit», lesquelles s'attachent à la manifestation immédiate de l'expérience vécue (Erlebnis) dans les gestes, paroles, attitudes et formes d'expression esthétique, religieuse et sociale, où le sens de l'expérience humaine prend visage. En prenant à son compte la distinction opérée par Johann Gustav Droysen (1808-1886) entre l'Erklären, l' «expliquer» qui prime dans le domaine des sciences de la nature et, par ailleurs, le Verstehen, le «comprendre» propre aux sciences de l'esprit, Dilthey va tenter de jeter les bases d'une "critique de la raison historique» (Dilthey 1992) de façon à conférer aux « sciences humaines» une validité épistémologique, certes nettement différenciée, mais à tout le moins comparable à celle dont jouissent les sciences de la nature. La ligne de démarcation passe par la distinction entre l'expérience au sens scientifique, l'Erfahrung qui est au fondement de la logique inductive prévalant dans les Naturwissenschaften et qui se distingue par la possibilité d'une répétition à volonté des résultats obtenus sous certaines conditions et, par ailleurs, l'expérience au sens d'Erlebnis, le monde du vécu, bref les modes d'apprentissage liés à des conditions variables, parfois purement circonstancielles et relatives à un tissu complexe de facteurs et de contingences qui ont pour horizon la dimension historique de cette expérience. L'Erlebnis comme expérience vécue, tributaire des aléas et des rares moments de félicité filant la trame de l'existence humaine, est donc ancrée dans la Lebenswelt, le "monde de la vie», et se projette dans un horizon de sens qui forme un "cercle herméneutique»: l'individu appréhende sa propre expérience vécue à la lumière d'un monde qui constitue l'horizon préalable de son expérience et ce monde ne s'ouvre à lui qu'à partir 
de sa compréhension préalable des données qu'il tire de cette expérience et du sens qu'il leur imprime au gré de ses diverses interprétations.

Pour Gadamer, le réquisit d'objectivité qui prévaut dans le domaine des sciences de la nature, qu'il n'est pas ici question de contester ou de justifier quant à sa valeur intrinsèque ou à la fécondité des protocoles et des procédures qui s'en autorisent, obstrue néanmoins la conscience en introduisant une «distance aliénante» (Verfremdung) qui oblitère le rapport fondamental d'appartenance (Zugehörigkeit) de l'homme au monde. Ce lien d'appartenance est noué de multiples façons, par exemple dans le domaine de l'esthétique qui fait appel au «tact» (Taktgefühl) et à l'intériorisation de la sensibilité (Einfühlung) qui modèlent la composante plastique des affects qui se prêtent alors plus volontiers à la formation intuitive et intellectuelle de la culture, de la Bildung. L'autoréflexion (Selbstreflexion) de la conscience rompue à l'aveu de finitude est aussi marquée par l'écoute attentive de la tradition qui prodigue un univers de référence au sein duquel la relation au monde se construit à la faveur d'aspirations nourrissant certaines attentes façonnées déjà par la «conscience de l'efficience historique» (wirkungsgeschichtliches Bewußtsein).

L'être conscient qui interroge les contenus qui façonnent la trame de son existence évolue dans un monde préstructuré par le travail effectif (Wirkung) de l'histoire qui détermine ses attentes et les divers modi intelligendi qui lui permettent d'actualiser son jugement, bref de concerter et de consolider le cours parfois rhapsodique de ses multiples délibérations sur la base de certains "préjugés» qui entrent dans la configuration de son «expectative», la notion de "préjugé» (Vorurteil) étant ici entendue dans le sens d'une pure structure d'anticipation (Vorgriff). Ainsi l'individu plongé dans ce que Husserl désignait comme das lebendige Gegenwart, le "Présent vivant», est-il du même coup ancré dans un monde qui le prédispose à juger de sa situation en fonction de l'interpénétration des horizons multiples du passé, du présent et de ce qu'il lui est loisible d'anticiper pour l'avenir, réalisant ce que Gadamer désigne à son tour comme une Horizontverschmelzung, une «fusion d'horizons".

En conséquence, la problématisation du « comprendre»(Verstehen) qui se saisit des données immédiates de l'expérience est médiatisée par une ouverture de sens préalable qui découle de cette «fusion d'horizons » enchevêtrés qui enveloppent et orientent notre rapport au texte et à la vive parole dans le dialogue. Le langage, conçu d'abord dans sa dimension dialogique, comme rapport à l'autre, aussi bien à l'altérité d'autrui qu'à celle qui émerge d'horizons passés ou plus récents, est la clef de voûte ou, si l'on peut dire, la clavis hermeneutica du questionnement élaboré par Gadamer. Pour lui, la vérité du langage ne s'accomplit pas dans la forme propositionnelle de l'énoncé mais dans la Verständigung, dans l'«entente» colloquiale qui lie dialogue et communauté d'interprétation. L'universalité au sens propre n'est pas l'«affaire» des propositions logiques, des constructions grammaticales et des règles syntaxiques, mais de la dimension d'ouverture qui s'engage et se déploie en ampleur et en intensité dans le Gespräch, le dialogue qui seul peut assurer une participation commune dans la production du sens. À la différence de la construction strictement propositionnelle (prédicative) de l'énoncé, l'espace logique de l'interlocution est régi, si je puis dire, par une dynamique non linéaire qui répond à la polysémie originelle investissant la langue d'une énergie propre qui est celle du dialogue, sa dimension "performative», où le langage se fait acte plutôt que simple relation d' "états de choses », corroborant la distinction opérée par Wilhelm von Humboldt lorsqu'il privilégie une conception de la langue, plus précisément de son exercice comme energeia ou énergie créatrice plutôt que simple ergon, une espèce d'artefact ou de produit figé et saturé sans son usage (1974: 183). L'expérience du langage revêt une telle importance pour Gadamer qu'il n'hésitera pas à affirmer que «le seul être qui puisse être compris est la langue ("Sein, das verstanden werden kann, ist Sprache»)».

Or ce parti pris en faveur de la dimension pragmatique et interlocutoire du langage n'est pas indifférent à la problématique de la traduction. Gadamer l'exprime en toutes lettres dans Vérité et méthode: "L'exemple du traducteur, dont la tâche est de franchir l'abîme qui sépare les langues, fait apparaître d'une façon particulièrement claire la relation mutuelle qui se déroule entre l'interprète et le texte, correspondant à la réciprocité de l'explication dans la conversation ». En fait, poursuit Gadamer, «la langue étrangère ne représente qu'une aggravation de la difficulté herméneutique, celle de l'»étrangèreté» et de son dépassement» (1996: 408-409). Gadamer corrobore ainsi l'intuition de Schleiermacher selon laquelle la différence entre l'acte de comprendre et l'acte de traduire n'est qu'une différence de degré. Non seulement la traduction se situe-t-elle dans l'horizon de la "problématicité» mise à découvert dans ce crépuscule interminable avec lequel finit par se confondre le passage à la modernité, mais elle représente un cas ou une situation exemplaire, paradigmatique, de la sollicitation herméneutique. Son insolubilité est au fondement même de sa dynamique. Pas question de conclure à un échec ou à une faillite, serait-elle de bon aloi, puisque le caractère aporétique, problématique, litigieux, de toute solution dans le négoce entre les langues est la condition même de son opération, ce pour quoi elle est sollicitée. Si, comme l'écrit Gadamer, «le traducteur a souvent une conscience 
douloureuse de la distance qui le sépare nécessairement de l'original», c'est que «sa fréquentation du texte comporte en elle-même quelque chose de l'effort que l'on fait pour s'entendre dans la conversation", et, dans ce cas comme dans bien d'autres où nous sommes compromis, mis à mal en raison de la responsabilité qui nous incombe face à autrui, force nous est de reconnaître, comme cela s'avère souvent dans la motion du "traduire», que «la distance qui sépare ce que veut dire l'autre de ce qu'on veut dire soi-même ne peut en définitive être abolie» (Gadamer 1996: 409).

Il y a fort à parier que nous n'aurions rien à nous dire si par quelque hasard désastreux une conscience uniforme pénétrée d'on ne sait trop quelle espèce de science infuse nous illuminait tout un chacun d'un savoir absolu qui nous dispenserait d'en discourir et de deviser de quoi que ce soit. L'impossibilité de la traduction simultanée est notre «chance» en fin de compte. Car elle nourrit nos attentes d'autant qu'elle les déçoit, nous obligeant à anticiper et à présumer toujours davantage d'un accord possible, mais sans doute irréalisable. C'est dire aussi que la dialectique de l'interprétation s'enracine dans la dialogique de l'interpellation, que son ethos, bref l'impératif dont procède son mandat est toujours réponse à un appel venu du tréfonds des âges où la forme humaine, tout entière concentrée dans la dynamique du verbe, a pris visage et s'est construite dans le nom. C'est en cela précisément que la traduction se trouve être de quelque façon le paradigme originel de la compréhension intersubjective. $\mathrm{Si}$, comme le suggère le fameux incipit johannique, "au commencement était le verbe», en ce temps improbable et certes affabulatoire devait aussi se trouver l'oreille qui assentit à l'interpellation, se laisse envahir par la séquence vibratoire qui sollicite son écoute. Dans une très belle conférence intitulée «Jusqu’à quel point la langue préforme-telle la pensée?», Gadamer nous invite à emprunter cette direction (1973: 68-69):

En laissant tout à fait de côté toutes les différences au sein de la transcription graphique, je dirais que tout écrit pour être compris a besoin d'une sorte d'élévation dans l'oreille intérieure. Pour la poésie et autres choses de ce genre, cela va de soi, mais pour la philosophie aussi $j$ 'ai l'habitude de dire à mes élèves: vous devez aiguiser votre oreille, vous devez savoir que quand vous prenez un mot dans votre bouche, vous n'avez pas utilisé un outil quelconque qui, s'il ne va pas, est jeté dans un coin, mais que vous êtes en réalité fixés dans une direction de pensée qui vient de loin et va beaucoup plus loin que vous. Il s'agit toujours d'une sorte de reconstitution que nous opérons. Je voudrais l'appeler traduction dans un sens très large. Lire c'est déjà traduire, et traduire c'est encore traduire. [...] Le processus de la traduction contient au fond tout le secret de la compréhension humaine du monde et de la communication sociale. La traduction est une unité indivisible d'anticipation implicite, de présomption du sens en général et de détermination explicite de ce qu'on avait ainsi présumé.

LAURENT LAMY Université de Montréal, Montréal, Canada c.lamy@sogetel.net

\section{NOTES}

* Cette étude est la version remaniée et augmentée d'une leçon prononcée dans le cadre du séminaire d'épistémologie que je dirigeais, à la session d'hiver 2002, au Département de linguistique et de traduction de l'Université de Montréal. Je tiens à remercier les participantes à ce séminaire, qui m'ont prodigué leur présence chaleureuse et attentive, mesdames Magali Cape, Elvia Rosa Castrillon, Adriana Lucia Diaz, Chantal Lemay, Annie Patenaude et Carole Poisson.

1. Jacques Bouveresse (1991: 21-22) résume fort bien les tenants et aboutissants de ce paradigme naissant: "La conviction essentielle des théologiens protestants qui sont considérés généralement comme les initiateurs de la tradition herméneutique est celle de l'auto suffisance (sola scriptura) et de l'auto-interprétabilité (sacra scriptura sui ipsius interpretes) de l'Écriture. Leur postulat de base est celui de l'accessibilité directe du sens du texte sacré ou encore, comme l'appelle Dilthey, de l'»autonomie normative de l'Écriture». Si les textes sacrés n'ont pas été compris, ce n'est pas parce qu'ils seraient intrinsèquement obscurs ou ambigus et ne deviendraient réellement intelligibles que grâce au complément indispensable apporté par la tradition et l'autorité des Pères de l'Église mais parce qu'ils ont été abordés avec des instruments exégétiques insuffisants ou inadéquats. La déclaration d'indépendance de l'exégèse protestante, qui a rendu possible la constitution d'une science herméneutique proprement dite, a pour corrélat implicite ou explicite la croyance à l'existence d'un sens déterminé du texte écrit, qui est à la disposition de l'interprète capable de l'effort de réappropriation nécessaire, un présupposé qui est, à bien des égards, le premier à être contesté par les représentants de l'herméneutique contemporaine».

2. Sauf pour saluer ici la parution d'un ouvrage tout à fait remarquable de Robert Wardy (2000), qui se livre à une réévaluation très judicieuse et nuancée de l'hypothèse Sapir-Whorf et de son ascendance chez Wilhelm von Humboldt à la faveur d'un examen minutieux du Ming Li T'an ( «'investigation de la théorie des noms»), une traduction en chinois du traité des Catégories d'Aristote datant du XVII ${ }^{\text {e }}$ siècle.

3. Je rappelle que c'est l'une des thèses fondamentales mises de l'avant par Walter Benjamin dans son fameux essai sur la «tâche du traducteur» (1997: 16), à savoir que «c'est uniquement à 
partir du moment où la vie est reconnue comme l'apanage de tout ce dont il y a histoire et qui ne se résume pas à en être le théâtre que son concept est investi dans son droit. Car c'est à partir de l'histoire, non point de la nature et, à plus forte raison, pas de notions aussi fluctuantes que l'âme et la sensation, que la sphère de la vie reçoit finalement sa pleine et entière détermination».

\section{RÉFÉRENCES}

BAnON, David (1987): La lecture infinie. Les voies de l'interprétation midrachique, Paris, Seuil.

Benjamin, W. (1986): Le concept de critique esthétique dans le romantisme allemand, trad. par Philippe Lacoue-Labarthe et Anne-Marie Lang, Paris, Flammarion.

Benjamin, W. (1997): «L'abandon du traducteur. Prolégomènes à la traduction des Tableaux parisiens de Charles Baudelaire», trad. et notes par Lamy, L. et A. Nouss, TTR 10-2, p. 13-62.

Berman, A. (1984): L'épreuve de l'étranger. Culture et traduction dans l'Allemagne romantique, Paris, Gallimard.

Berner, C. (1995): La philosophie de Schleiermacher. Herméneutique, dialectique, éthique, Paris, Cerf.

Berner, C. (2001): «Aimer comprendre. Recherche sur les fondements éthiques de l'herméneutique de Schleiermacher", Revue de Métaphysique et de Morale 1, p. 63-84.

Billeter, J.-F. (2002): Leçons sur Tchouang-tseu, Paris, Allia.

Bouveresse, Jacques (1991): Herméneutique et linguistique, Combès, Éd. de l'éclat.

BOYARIN, D. (1994): Intertextuality and the Reading of the Midrash, Bloomington et Indianapolis, Indiana University Press.

Budick, S. and W. Iser (1996): The Translatability of Cultures: Figurations of the Space Between, Stanford, Stanford University Press.

Delpla, I. (2001): Quine, Davidson. Le principe de charité, Paris, PUF.

Dilthey, W. (1992): Euvres I: Critique de la raison historique. Introduction aux sciences de l'esprit, Paris, Cerf.

Foucault, M. (1966): Les mots et les choses. Une archéologie des sciences humaines, Paris, Gallimard.

GadAmer, H.-G. (1996) : Vérité et méthode. Les grandes lignes d'une herméneutique philosophique, trad. par Fruchon, P., Grondin, J. et G. MerLIO, Paris, Seuil.

Gadamer, H.-G. (1996a): La philosophie herméneutique, avant-propos, trad. et notes de Grondin, J., Paris, PUF.

Gadamer, H.-G. (1973) : «Jusqu'à quel point la langue préforme-t-elle la pensée?», in CAsTelli, C. (éd.), Démythisation et idéologie, Paris, Aubier Montaigne, p. 63-70.

Greisch, J. (2000) : Le cogito herméneutique. L'herméneutique philosophique et l'héritage cartésien, Pris, Vrin.
Hölderlin, F. (1967): Euvres, édition de Philippe Jaccottet, Paris, Gallimard.

Нumboldt, W. von (1974): Introduction à l'œuvre sur le Kavi et autres essais, trad. et introduction de Pierre Caussat, Paris, Seuil.

Idel, M. (1998): "Preface», Betty Rojtman, Black Fire on White Fire. An Essay on Jewish Hermeneutics, from Midrash to Kabbalah, trad. par Steven Rendall, Berkeley, University of California Press, p. ix-xii.

Lacoue-Labarthe, P. et J.-L. Nancy (1978): L'Absolu littéraire. Théorie de la littérature du romantisme allemand, Paris, Seuil.

Laks, A. et A. Neschke (1990): La naissance du paradigme herméneutique. Schleiermacher, Humboldt, Boeckh, Droysen, Lille, Presses universitaires de Lille.

LAugier, S. (2001): "Charité, traduction radicale et prélogicité", Revue de Métaphysique et de Morale 1, p. 63-84.

Neschke, A. (1990): «Matériaux pour une approche philologique de l'herméneutique de Schleiermacher» et «Le texte de Platon entre Friedrich August Wolf (1759-1824) et Friedrich Schleiermacher (1767-1834)», in LaKs, A. et A. NesCHKE (1990), p. 33-35 et 257-258.

Pyм, A. (1997): Pour une éthique du traducteur, Arras/Ottawa, Artois Presses Université/Presses de l'Université d'Ottawa.

Quine, W.V. O. (1969): Word and Object, Cambridge, Mass., MIT Press.

Rojtman, B. (1986): Feu noir sur feu blanc. Essai sur l'herméneutique juive, Lagrasse, Verdier.

Schlegel, F. (1996): "Philosophie de la philologie», in Thouard, D. (1996), fragment 18, p. 214.

Schleiermacher, F. (1995): Herméneutique, trad. de Berner, C., Paris/Lille, Cerf/Presses universitaires de Lille.

Schleiermacher, F. (1996): "Sur la Lucinde de Schlegel», "Sur les caractéristiques et critiques de Schlegel», "Fragments sur le langage et l'herméneutique», "Sur le concept et la division de la critique philologique», trad. par THOUARD, D., in Thouard, D. (1996), p. 71-77, 101-109, 279-285 et 315-328.

Schleiermacher, F. (1997): Dialectique, trad. par Berner, C. et D. Thouard, avec la collaboration de J.-M. Tétaz, Paris/Genève/Québec, Cerf/Labor et Fides/Presses de l'Université Laval.

Schleiermacher, F. (1999): Des différentes méthodes du traduire et autre texte, éd. bilingue allemandfrançais, trad. par Berman, A. et C. Berner, Paris, Seuil.

SChleiermacher, F. (2004): Introductions aux dialogues de Platon (1804-1828), Leçons d'histoire de la philosophie (1819-1823), suivies des textes de Friedrich Schlegel relatifs à Platon, trad. et introduction par Richard, M.-D., Paris, Cerf.

Szondi, P. (1975) : «L'herméneutique de Schleiermacher", in Poésie et poétique de l'idéalisme allemand, trad. dirigée par Bollack, J. avec la 
collaboration de Barbara Cassin, Isabelle Michot, Jacques Michot, Helen Stierlin, Paris, Minuit, p. 291-315.

Szond, P. (1989): Introduction à l'herméneutique littéraire. De Chladenius à Schleiermacher, trad. par Bollack, M., Paris, Cerf.

Thouard, D. (1996): Critique et herméneutique dans le premier romantisme allemand, textes traduits et annotés par Thouard, D., Villeneuve-d'Ascq, Presses universitaires du Septentrion.

Thouard, D. (2002): «Qu'est-ce qu’une "herméneutique critique"? ", Methodos, 2: «L'esprit. Mind/ Geist» : http://methodos.revues.org/document 100.html.
Todonov, T. (1981): Mikhaïl Bakhtine. Le principe dialogique, suivi de Écrits du Cercle de Bakhtine, Paris, Seuil.

VAN EYNDE, L. (1997): Introduction au romatisme d'Iéna. Friedrich Schlegel et l'Athenäum, Bruxelles, Ousia.

Wardy, R. (2000): Aristotle in China: Language, Categories, and Translation, New York et Cambridge, Cambridge University Press.

Wittgenstein, L. (1982): Notes sur l'«expérience privée» et les «sense data», trad. par Rigal, E., Mauvezin, Trans Europ Repress. 